\title{
7 O EFEITO MODERADOR DA SATISFAÇÃO COM A VIDA NA ASSOCIAÇÃO ENTRE A QUALIDADE DA RELAÇÃO PAIS/FILHOS(AS) E DEPRESSÃO NA ADOLESCÊNCIA ${ }^{1}$
}

| Bárbara Salgado Costa ${ }^{2}$; Ana Paula Matos ${ }^{3}$; José Joaquim Costa ${ }^{4}$ |

\section{RESUMO}

CONTEXTO: A depressão apresenta um caráter duradouro e relaciona-se com perdas funcionais significativas e com uma redução da qualidade de vida a longo prazo. Existem vários fatores que vulnerabilizam o ser humano ao seu desenvolvimento, nos quais se incluem os relacionamentos familiares de baixa qualidade caraterizados por elevados níveis de conflito e a baixa satisfação com a vida.

OBJETIVO(S): Estudar como preditores da depressão na adolescência a qualidade da relação pais/filhos(as) e a satisfação com a vida e explorar o efeito moderador da satisfação com a vida, na associação entre a qualidade da relação entre pais/filhos(as) avaliadas num primeiro momento e a depressão na adolescência avaliada no segundo momento (seis meses depois).

METODOLOGIA: Numa amostra comunitária de 534 adolescentes, com idades entre os 13 e 17 anos foram aplicados questionários e uma entrevista em dois momentos de avaliação, com um intervalo de 6 meses.

RESULTADOS: Os adolescentes que avaliaram a sua satisfação com a vida como boa, revelaram menos sintomatologia depressiva do que aqueles que avaliaram como razoável ou baixa, mesmo existindo perceção de conflito mais elevada no relacionamento com a mãe.

CONCLUSÕES: A satisfação com a vida avaliada como boa pode funcionar como fator de proteção no desenvolvimento de sintomas depressivos, perante níveis altos de conflito no relacionamento com a mãe. Enfatiza-se, assim, a inclusão e avaliação de construtos oriundos da psicologia positiva em programas preventivos da depressão na adolescência.

PALAVRAS-CHAVE: Depressão; Adolescente; Relações familiares; Satisfação pessoal

\section{RESUMEN}

"El efecto moderador de la satisfacción con la vida en la asociación entre la calidad de la relación padres/hijos(as) y depresión en la adolescencia ${ }^{1 "}$

ANTECEDENTES: La depresión tiene un carácter a largo plazo y está relacionada con importantes pérdidas funcionales y una reducción en la calidad de vida a largo plazo. Hay varios factores que hacen que el ser humano sea vulnerable a la depresión, incluidas las relaciones familiares de baja calidad caracterizadas por altos niveles de conflicto y baja satisfacción con la vida. OBJETIVO: Estudiar la calidad de la relación padres/hijos y la satisfacción con la vida como predictores de la depresión en la adolescencia y explorar el efecto moderador de la satisfacción vital en la asociación entre la calidad de la relación entre padres/hijos evaluados en el primer momento y la depresión en la adolescencia evaluada en el segundo momento (seis meses después).

METODOLOGIA: En una muestra comunitaria de 534 adolescentes, de entre 13 y 17 años, se aplicaron cuestionarios y se realizó una entrevista en dos momentos de evaluación, con un intervalo de 6 meses.

RESULTADOS: Los adolescentes que evaluaron su satisfacción con la vida como buena mostraron menos sintomatología depresiva que aquellos que calificaron como razonable o baja, incluso existiendo una percepción de un mayor conflicto en la relación con la madre.

CONCLUSIONES: La satisfacción con la vida evaluada como buena puede actuar como un factor protector en el desarrollo de síntomas depresivos, ante niveles altos de conflicto en la relación con la madre. Se enfatiza la inclusión y evaluación de conceptos de la psicología positiva en los programas preventivos para la depresión en la adolescencia.

DESCRIPTORES: Depresión; Adolescente; Relaciones familiares; Satisfacción personal

\begin{abstract}
"The moderating effect of life satisfaction in the association between the quality of the parents/children relationship and depression in adolescence ${ }^{1 "}$

BACKGROUND: Depression has a long-term character and is related to significant functional losses and a reduction in the long-term quality of life. There are several factors that make the human being vulnerable to depression, including low quality family relationships characterized by high levels of conflict and low life satisfaction.

AIM: To study the quality of the parents/children relationship and life satisfaction as predictors of depression in adolescence and to explore the moderating effect of life satisfaction in the association between the quality of the relationship between the parents/children evaluated in the first moment and the depression in the adolescence evaluated in the second moment (six months later).

METHODS: In a community sample of 534 adolescents, aged 13 to 17 years, questionnaires were applied and an interview was carried out in two evaluation moments, with an interval of 6 months.

RESULTS: Adolescents who evaluated their life satisfaction as good showed less depressive symptomatology than those who assessed as reasonable or low, even though there was a perception of a higher conflict in the relationship with the mother.

CONCLUSIONS: Life satisfaction evaluated as good may act as a protective factor in the development of depressive symptoms, when facing high levels of conflict in the relationship with mother. Emphasis is thus given to the inclusion and evaluation of constructs from positive psychology in preventive programs for depression in adolescence.
\end{abstract}

\section{KEYWORDS: Depression; Adolescent; Family relations; Per- sonal satisfaction}

Submetido em 30-12-2017 Aceite em 21-03-2018

1 Este artigo foi extraído da Dissertação de Mestrado da primeira autora, sobre o tema: "Um Estudo Longitudinal sobre o Efeito Moderador da Satisfação com a vida na associação entre a Qualidade da Relação Pais/Filhos(as) e Depressão na Adolescência” (2017) - apresentada na Faculdade de Psicologia e Ciências da Educação da Universidade de Coimbra.

2 Mestre em Psicologia, barbaracostapsi@hotmail.com

3 Doutorada em Psicologia; Investigadora do Centro de Investigação do Núcleo de Estudos e Intervenção Cognitivo-Comportamental; Professora Associada na Faculdade de Psicologia e de Ciências da Educação da Universidade de Coimbra, Rua Colégio Novo, 3000-115 Coimbra, Portugal, apmatos@fpce.uc.pt

4 Doutorado em Psicologia; Investigador do Centro de Investigação do Núcleo de Estudos e Intervenção Cognitivo-Comportamental; Professor Auxiliar na Faculdade de Psicologia e de Ciências da Educação da Universidade de Coimbra, 3000-115 Coimbra, Portugal, jjcosta@fpce.uc.pt

Citação: Costa, B. S., Matos, A. P., \& Costa, J. J. (2018). O efeito moderador da satisfação com a vida na associação entre a qualidade da relação pais/filhos(as) e depressão na adolescência. Revista Portuguesa de Enfermagem de Saúde Mental (Spe. 6), 46-51. doi: 10.19131/rpesm.0212 


\section{INTRODUÇÃO}

Durante a adolescência ocorrem intensas mudanças desenvolvimentais, que marcam a transição entre a infância e a idade adulta. Assim, esta etapa é muito importante para construir a identidade do indivíduo e para a busca da sua independência tão desejada (Cicchetti \& Toth, 2009). A qualidade da relação que os adultos (e.g., pais e professores) estabelecem com o adolescente, a atenção que lhe dão, são decisivas para a edificação da sua identidade. $\mathrm{O}$ adolescente vai enfrentar a necessidade de se redefinir em relação ao seu corpo sexuado, à sua identidade psíquica e ao seu meio, e particularmente em relação aos seus pais (Brito, 2011). A depressão na adolescência nem sempre foi reconhecida como uma perturbação psiquiátrica, mas atualmente sabe-se que as perturbações de humor (perturbação depressiva major e perturbação distimica), em especial a depressão, são das mais prevalentes nos adolescentes (Arnarson \& Craighead, 2009). As perturbações de humor, das quais a depressão faz parte, caracterizam-se por mudanças acentuadas nos estados de alegria e tristeza, bem como na motivação para realizar a maior parte das atividades quotidianas e na energia necessária para as fazer (American Psychiatric Association, 2013). Embora a adolescência seja uma época em que os jovens se autonomizam dos progenitores, a relação com eles é essencial para a saúde psicológica (Kenny, Dooley \& Fitzgerald, 2013). Fatores como o controlo psicológico, o grau de conflito com os pais, e a rejeição dos progenitores estão associados à depressão. A má qualidade do ambiente familiar, os altos níveis de conflito familiar e os baixos níveis de apoio familiar podem predizer o surgimento e desenvolvimento de episódios depressivos nos adolescentes (Queen, Stewart, Ehrenreich-May \& Pincus, 2012). Num estudo transversal com uma amostra de adolescentes portugueses, verificou-se que níveis altos de conflito são preditores de mais sintomas depressivos (Brito, Matos, Pinheiro e Monteiro, 2015).

A satisfação com a vida assim como os afetos positivos, componentes do bem-estar subjetivo, predizem saúde e longevidade. O bem-estar subjetivo refere-se a avaliações que os indivíduos fazem sobre as suas vidas, que podem ser um julgamento cognitivo, como a satisfação com a vida, ou avaliações baseadas em sentimentos e emoções (Diener \& Chan, 2011). Assim, o bem-estar subjetivo é uma categoria heterogénea, que integra emoções agradáveis e emoções negativas e uma dimensão cognitiva (satisfação com a vida).
No que se refere ao bem-estar psicológico e à satisfação com a vida, a boa comunicação com os pais e com os amigos surge evidenciada no bem-estar dos adolescentes portugueses. A satisfação com a vida revela ser uma variável importante durante a adolescência (Çivitci \& Çivitci, 2009).

Gledhill \& Garralda (2011) destacaram o papel de acontecimentos de vida positivos na redução da depressão, mencionando a importância de programar atividades agradáveis ou gratificantes.

Em Portugal, numa investigação com uma amostra de 400 sujeitos, encontrou-se que os adolescentes que percecionam melhor ambiente familiar e que têm uma vinculação mais segura aos pais reportam maior satisfação com a vida (Machado e Fonseca, 2009).

Em 2008, Antaramian, Huebner \& Valois constataram que a satisfação com a vida é menor entre os jovens que experimentam níveis mais elevados de conflito e de desacordo com os pais. Num estudo transversal (Brito et al., 2015) verificou-se que pontuações baixas no fator conflito com o pai, interagindo com níveis altos de satisfação com a vida, podem funcionar como fatores de proteção da sintomatologia depressiva.

\section{METODOLOGIA}

Este estudo longitudinal insere-se no projeto de investigação "Prevenção da Depressão em Adolescentes Portugueses: Estudo da Eficácia de uma Intervenção com Adolescentes e Pais" (projeto com a referência: PTDC/MHC-PC L/4824/2012), financiado pela Fundação para a Ciência e Tecnologia (FCT) e pela Realan Foundation (Estados Unidos da América). O presente estudo pretendeu estudar a qualidade da relação pais/filhos e a satisfação com a vida dos adolescentes, enquanto preditores de depressão no adolescente e, analisar o efeito moderador da satisfação com a vida na associação entre a qualidade da relação pais e filhos(as) e a sintomatologia depressiva.

A amostra deste estudo foi constituída por 534 adolescentes. A média de idade da amostra é de 14,45 anos $(\mathrm{DP}=.89)$. A maioria são raparigas $(61 \%)$. Os inquiridos pertencem predominantemente a um nível socioeconómico baixo (51.5\%). Os restantes participantes pertencem a um nível socioeconómico médio (35.4\%) e elevado (13.1\%). A maioria tem os pais casados $(74.3 \%)$.

Recorreu-se a uma amostra comunitária, recolhida em escolas públicas e privadas, da região Centro de Portugal. 
Os adolescentes e respetivos encarregados de educação preencheram um consentimento informado. E o estudo foi autorizado pela Comissão Nacional de Proteção de Dados (CNPD) e pela Direção Geral de Inovação e Desenvolvimento Curricular (DGIDC).

Para a colheita de dados foram utilizados os seguintes instrumentos: Children Depression Inventory (CDI) (versão portuguesa: Marujo, 1994) para medir a sintomatologia depressiva, Inventory of the Quality of Interpersonal Relations (IQRI) (versão portuguesa para adolescentes: Marques, Matos, e Pinheiro, 2014; Marques, Pinheiro, Matos, e Marques, 2015), para avaliar a qualidade das relações interpessoais, e a Adolescent - Longitudinal Interval Follow-up Evalution (A-LIFE) (versão portuguesa: Matos e Costa, 2011). O CDI é composto por 27 itens, divididos em cinco fatores: anedonia, autoestima negativa, humor negativo, ineficácia e problemas interpessoais. A resposta a cada item tem três alternativas numa escala de 0 (ausência de sintoma) a 2 pontos (sintoma grave). É solicitado que selecione o valor que melhor descreve os seus sentimentos durante as "duas últimas semanas". A pontuação total varia entre 0 e 54 pontos. Considerou-se apenas a pontuação total da escala, tendo em conta a estrutura unifatorial encontrada para a população portuguesa. $\mathrm{O}$ valor de alfa de Cronbach para a pontuação do CDI total foi de .90 , revelando boa consistência interna. O IQRI é constituído por 25 itens, organizados em três subescalas, (perceção de suporte, de profundidade e de conflito), avaliadas pelo sujeito no relacionamento com a mãe e o pai. No presente estudo analisámos apenas a dimensão conflito. Os itens são cotados numa escala tipo Likert de 1 a 4 e quanto maior for a pontuação obtida, mais presente está essa dimensão. Na presente investigação o IQRI mostrou boa consistência interna para todos os fatores (valores de alfa de Cronbach entre .80 e .91). A A-LIFE dividese em três secções gerais, Psicopatologia, Funcionamento Psicossocial e Gravidade Geral da doença. Para além disso, avalia também a Satisfação Global com a Vida, isto é, o contentamento da pessoa com a relação com a família, com os amigos, atividades que desenvolve, trabalho, situação económica e atividades sexuais. É solicitado ao adolescente que tenha em conta a "pior semana de cada mês para os últimos seis meses". A pontuação é analisada numa escala tipo Likert de 1 a 5, sendo 1) " muito bom" e 5) "muito pobre".

Previamente à realização das análises estatísticas, a variável satisfação com a vida foi agrupada em três níveis (muito bom, bom e razoável ou baixo).
Como as classes correspondentes à baixa satisfação com a vida (satisfação pobre e muito pobre), tinham apenas no seu total nove adolescentes, optou-se por agrupá-las na classe da satisfação razoável, passando a ser designada por classe de satisfação "razoável ou baixa”. A variável moderadora, sendo categorial foi considerada variável dummy. Criaram-se dois termos de interação, para as variáveis conflito com pai e mãe. Primeiramente selecionou-se a variável preditora (fatores do IQRI), de seguida colocou-se a variável moderadora (variáveis dummy da satisfação com a vida), e, para finalizar, a interação entre as duas.

\section{RESULTADOS}

Uma análise de regressão múltipla hierárquica foi conduzida com vista a explorar a possibilidade de existir um efeito moderador da satisfação com a vida, na associação entre a qualidade da relação pais/filhos(as) avaliada no primeiro momento e a sintomatologia depressiva, avaliada no segundo momento, após seis meses. Apenas a interação com a variável conflito no relacionamento com a mãe foi significativa, pelo que apresentamos apenas os resultados relativos à mesma. Constatou-se, através dos coeficientes de regressão $(\beta)$, que a dimensão conflito na relação com a mãe produziu um efeito moderador significativo quando em interação com o nível bom da satisfação com a vida $(\beta=-.113, \mathrm{p}=.015)$ (Tabela 1$)$.

Tabela 1 - Coeficientes de regressão das três etapas produzidas por uma regressão múltipla hierárquica com o conflito da mãe, satisfação com a vida e o termo de interação na predição de sintomatologia depressiva na amostra total $(\mathrm{N}=534)$

\begin{tabular}{|l|l|c|c|c|c|c|}
\hline Mod. & Preditores & $\mathrm{R}^{2}$ & $\mathrm{~F}$ & $\beta$ & $\mathrm{t}$ & $\mathrm{p}$ \\
\hline 1 & Mãe conflito & .173 & 110.981 & .415 & 10.535 & .000 \\
\hline 2 & Mãe conflito & .279 & 68.220 & .323 & ${ }^{*} 8.403$ & .000 \\
\cline { 2 - 7 } & $\begin{array}{l}\text { Muito boa satis- } \\
\text { fação com a vida }\end{array}$ & & & -.379 & -7.915 & .000 \\
\cline { 2 - 7 } & $\begin{array}{l}\text { Boa satisfação } \\
\text { com a vida }\end{array}$ & & & -.379 & -7.987 & .000 \\
\hline 3 & Mãe conflito & .287 & 42.487 & .313 & $\star 8.151$ & .000 \\
\cline { 2 - 7 } & $\begin{array}{l}\text { Muito boa satis- } \\
\text { fação com a vida }\end{array}$ & & & -.350 & -7.062 & .000 \\
\cline { 2 - 7 } & $\begin{array}{l}\text { Boa satisfação } \\
\text { com a vida }\end{array}$ & & & -.341 & -6.863 & .000 \\
\cline { 2 - 7 } & $\begin{array}{l}\text { Mãe conflito } \\
\text { muito boa satis- } \\
\text { fação com a vida }\end{array}$ & & & -.080 & -1.747 & .081 \\
\cline { 2 - 7 } & $\begin{array}{l}\text { Mãe conflito* } \\
\text { boa satisfação } \\
\text { com a vida }\end{array}$ & & & -.113 & -2.437 & .015 \\
\hline
\end{tabular}


Quando consideramos como preditor o fator conflito na relação com a mãe, obtêm-se modelos estatisticamente significativos em todos os passos. A interação entre as variáveis preditora e moderadora produziu um coeficiente de determinação de $28.7 \%$. Os dados indicam que o declive que se prevê nos sintomas depressivos de acordo com o conflito percecionado pelos adolescentes no relacionamento com a mãe difere significativamente de acordo com os valores altos ou baixos da satisfação com a vida. Com o intuito de interpretar e compreender melhor a interação significativa, elaborou-se um gráfico (Gráfico 1).

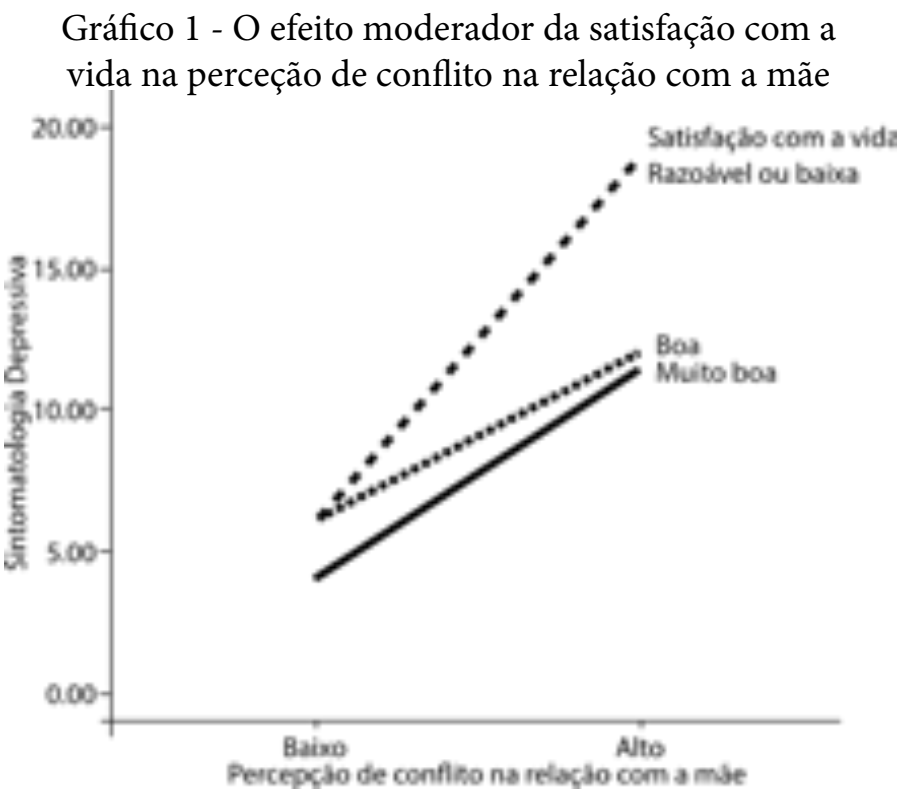

Observa-se que níveis mais elevados de conflito na relação com a mãe e níveis mais baixos na satisfação com a vida estão associados a maior sintomatologia depressiva. No que toca ao termo de interação, verifica-se que quando a perceção de conflito com a mãe é alta no primeiro momento de avaliação, o nível bom da satisfação com a vida, comparativamente ao nível razoável ou baixo, no primeiro momento de avaliação, funciona como fator protetor do desenvolvimento de sintomatologia depressiva no segundo momento de avaliação, reportando estes adolescentes menos depressão. Já quando a perceção de conflito com a mãe é alta, são os adolescentes que têm muito boa satisfação com a vida que apresentam menos sintomatologia depressiva.

\section{DISCUSSÃO}

A perceção de elevados níveis de conflito no relacionamento com a mãe, avaliada no primeiro momento, revelou-se preditora de desenvolvimento de sintomatologia depressiva nos adolescentes, avaliada no segundo momento.
Este dado é congruente com os resultados encontrados por Queen, Stewart, Ehrenreich-May \& Pincus, 2012.

No presente estudo, verificou-se uma interação significativa entre a satisfação com a vida avaliada como boa e a perceção de conflito no relacionamento com a mãe na predição de sintomatologia depressiva. Os nossos dados mostram assim, que o conflito com a mãe funciona como um fator de risco para o desenvolvimento de sintomas depressivos e que, diante de uma satisfação com a vida avaliada como boa, estes sintomas tendem a ser menores. Estes resultados divergem do estudo transversal de Brito et al. (2015), no qual se encontrou uma interação significativa, mas entre a perceção de conflito com o pai e a satisfação com a vida na predição de sintomatologia depressiva. O presente estudo apresenta algumas limitações. Os resultados foram obtidos com base numa amostra comunitária, não abrangendo níveis elevados de sintomatologia depressiva nem níveis muito baixos de satisfação com a vida. Numa amostra clínica poderse-ão obter resultados diferentes.

Algumas variáveis não foram controladas, como a desejabilidade social, desmotivação e negação/minimização, o que pode ter influenciado as respostas dos participantes. Futuramente, deverá recorrer-se a amostras clínicas e também a amostras mais representativas da população geral portuguesa, para possibilitar uma melhor compreensão sobre o desenvolvimento psicológico adaptativo e mal adaptativo durante a adolescência.

\section{CONCLUSÃO}

Este estudo longitudinal mostrou que um nível bom de satisfação com a vida pode funcionar como fator de proteção no desenvolvimento de sintomas depressivos sobretudo em adolescentes com uma relação conflituosa com a mãe. Seligman (2011) tem salientado também que componentes do bem-estar, como a satisfação com a vida, previnem a depressão. Assim, encontrou-se que mesmo perante relações familiares de baixa qualidade, existem fatores que promovem a saúde mental, nomeadamente a satisfação com a vida, revelando-se importante olhar para a saúde mental como, não só a ausência de sintomas negativos, mas, também a presença de aspetos positivos. Este estudo vem contribuir para dar suporte à importância da satisfação com vida num desenvolvimento de saúde mental. 
Comprovou-se ainda, neste estudo, que a qualidade da relação pais/filhos(as) continua a ser importante para prevenir sintomatologia depressiva na fase da adolescência, pelo que a manutenção de uma relação com qualidade não deve ser descurada pelos progenitores.

\section{IMPLICAÇÕES PARA A PRÁTICA CLÍNICA}

A investigação traz contribuições para a prática clínica, pois evidencia a necessidade de intervir, junto dos adolescentes e dos seus pais, no sentido de melhorar relações que se estabelecem entre ambos. O que pode funcionar como prevenção do aparecimento de depressão nesta etapa da vida, conhecida pelos desafios particulares que acarreta. A integração nos programas de prevenção da depressão na adolescência da avaliação da qualidade da relação com os pais e da satisfação com a vida, de forma a aprofundar o conhecimento sobre os fatores de vulnerabilidade e de proteção ao desenvolvimento da sintomatologia depressiva, permitirá uma maior eficácia na prevenção e tratamento da depressão. Realça-se também a importância da inclusão de componentes associadas à satisfação com a vida nos programas de prevenção da depressão na adolescência, tal como já foi efetuado em alguns programas (Reiter \& Wilz, 2015). No entanto, a revisão da literatura efetuada mostra que ainda são poucas as intervenções dirigidas para a prevenção de depressão que tenham em conta construtos oriundos da psicologia positiva (Singhal, Manjula \& Sagar, 2015; McCarty, Violette \& McCauley, 2010).

\section{REFERÊNCIAS BIBLIOGRÁFICAS}

American Psychiatric Association. (2013). Diagnostic and statistical manual of mental disorders: DSM-5 ( $5^{\mathrm{a}}$ ed.). Washington, D.C: APA.

Antaramian, S. P., Huebner, E. S., \& Valois, R. F. (2008). Adolescent life satisfaction. Applied Psychology, 57 (s1), 112-126. Doi: 10.1111/j.1464-0597.2008. 00357.x

Arnarson, E., \& Craighead, W. (2009). Prevention of depression among Icelandic adolescents. Behavior Research and Therapy, 47 (7), 577-585. Doi: 10.1016/j. brat.2009.03.011.

Brito, I. (2011). Ansiedade e depressão na adolescência. Revista Portuguesa Clínica Geral, 27, 208-214.
Brito, J., Matos, A. P., Pinheiro, M. R., \& Monteiro, S. (2015). Quality of interpersonal relationships and depression in adolescence: Psychosocial functioning moderating effect. The European Proceedings of Social \& Behavioural Sciences, 81-94. Doi: 10.15405/ epsbs.2015.0.8.8

Cicchetti, D., \& Toth, S. (2009). A developmental psychopathology perspective on adolescent depression. In S. Nolen-Hoeksema, \& L. Hilt (Eds.), Handbook of Depression in Adolescents (pp. 3-32). New York, NY: Routledge.

Çivitci, N., \& Çivitci, A. (2009). Self-esteem as mediator and moderator of the relationship between loneliness and life satisfaction in adolescents. Personality and Individual Differences, 47 (8), 954-958. Disponível em: https://www.sciencedirect.com/science/article/ pii/S0191886909003419

Diener, E., \& Chan, M. Y. (2011). Happy people live longer: Subjective well-being contributes to health and longevity. Applied Psychology: Health and Well-Being, 3 (1), 1-43. Disponível em: https://onlinelibrary. wiley.com/doi/abs/10.1111/j.1758-0854.2010.01045.x

Gledhill, J., \& Garralda, M. E. (2011). The short-term outcome of depressive disorder in adolescents attending primary care: a cohort study. Social Psychiatry and Psychiatric Epidemiology, 46 (10), 993-1002. Doi: 10.1007/s00127-0100271-6.

Kenny, R., Dooley, B., \& Fitzgerald, A. (2013). Interpersonal relationships and emotional distress in adolescence. Journal of Adolescence, 36 (2), 351-360. Doi: 10.1016/j.adolescence.2012.12.005.

Machado, T. S., \& Fonseca, A. C. (2009). Desenvolvimento adaptativo em jovens portugueses: será significativa a relação com os pais. INFAD Revista de Psicologia, 1 (3), 461-468. Disponível em: http://www. redalyc.org/articulo.oa?id=349832322051

Marques, D., Matos, A. P., \& Pinheiro, M. D. R. (2014). Estudo da estrutura fatorial da versão mãe do IQRI para adolescentes. Psicologia, Saúde \& Doenças, 15 (1), 233-243. Disponível em: http://www. scielo.mec.pt/scielo.php?script=sci_arttext\&pid $=$ S1645-00862014000100019 
